PROCEEDINGS OF THE

AMERICAN MATHEMATICAL SOCIETY

Volume 140, Number 3, March 2012, Pages 1045-1052

S 0002-9939(2011)10965-1

Article electronically published on June 29, 2011

\title{
ON AN INCLUSION OF THE ESSENTIAL SPECTRUM OF LAPLACIANS UNDER NON-COMPACT CHANGE OF METRIC
}

\author{
JUN MASAMUNE
}

(Communicated by Matthew J. Gursky)

\begin{abstract}
The stability of essential self-adjointness and an inclusion of the essential spectra of Laplacians under the change of a Riemannian metric on a subset $K$ of $M$ are proved. The set $K$ may have infinite volume measured with the new metric, and its completion may contain a singular set such as the fractal set, to which the metric is not extendable.
\end{abstract}

\section{INTRODUCTION}

Let $(M, g)$ be a connected smooth Riemannian manifold without boundary. The Laplacian $\Delta$ of $g$ is called essentially self-adjoint if it has the unique self-adjoint extension $\bar{\Delta}$. In [4], Furutani showed that if $\Delta$ with the domain $C_{0}^{\infty}(M)$ is essentially self-adjoint and if $g$ is changed on a compact set $K \subset M$ to another smooth metric $g^{\prime}$ on $M$, then the Laplacian $\Delta^{\prime}$ of $g^{\prime}$ with the domain $C_{0}^{\infty}(M)$ is essentially self-adjoint; and the essential spectra are stable under this change. In particular, the second result forms a strong contrast to the behavior of the eigenvalues, since eigenvalues change continuously with the perturbation of the metric in a certain way (see e.g. [1]).

Needless to say, there are many important Riemannian manifolds with singularity, by which we mean that $g$ does not extend to the Cauchy boundary (the difference between the completion of $M$ and $M$ ), such as algebraic varieties, cone manifolds, edge manifolds, Riemannian orbifolds. In general, the analysis on such a singular space is complicated, and one of the methods to overcome the difficulties is to modify the singularity to a simpler one by the perturbation of the Riemannian metric. The crucial steps in this process is to study the stability of the essential self-adjointness of the Laplacian and to understand the behavior of its spectral structure under the perturbation.

Motivated by these facts, we extend Furutani's theorem to more general $K$ so that $K$ is not compact and its completion $\bar{K}$ includes the singular set. In this

Received by the editors March 30, 2010 and, in revised form, September 23, 2010 and December 8,2010 .

2010 Mathematics Subject Classification. Primary 58J50; Secondary 47B25.

Key words and phrases. Essential self-adjointness, incomplete manifolds, essential spectrum, perturbations.

(C)2011 American Mathematical Society Reverts to public domain 28 years from publication 
setting, the natural domain $D(\Delta)$ for the Laplacian is the following:

$$
\left\{\begin{array}{l}
D(\nabla)=\left\{u \in C^{\infty} \cap L^{2}: \nabla u \in L^{2}\right\}, \\
D(\operatorname{div})=\left\{X \in C^{\infty} \cap L^{2}: \operatorname{div} X \in L^{2}\right\}, \\
D(\Delta)=\{u \in D(\nabla): \nabla u \in D(\operatorname{div})\} .
\end{array}\right.
$$

(We suppress $M$ and the Riemann measure $d \mu_{g}$ for the sake of simplicity.) Indeed, if the Cauchy boundary $\partial_{C} M$ is almost polar, namely,

$$
\operatorname{Cap}\left(\partial_{C} M\right)=0
$$

(see Section 2 for the definition and see also e.g. 3]), then $M$ has negligible boundary [9], and by the Gaffney theorem [6], $\Delta$ is essentially self-adjoint. Throughout the article, we assume that the Laplacians have the domain defined in (1). The following is our main result:

Theorem 1. Let $g$ and $g^{\prime}$ be Riemannian metrics on $M$ such that $g=g^{\prime}$ outside a subset $K$ of $M$. If $\Delta$ is essentially self-adjoint in $L^{2}$ and the Cauchy boundary of $K$ with respect to $g^{\prime}$ is almost polar, then $\Delta^{\prime}$ is essentially self-adjoint in $L^{2}\left(M ; d \mu_{g^{\prime}}\right)$. Additionally, if there is a function $\chi$ on $M$ satisfying

$$
\nabla \chi \in L^{\infty}, \Delta \chi \in L^{\infty}, \text { and }\left.\chi\right|_{K}=1,
$$

where $\nabla$ is the gradient of $g$, and the inclusion

$$
H_{0}^{1}\left(N ; d \mu_{g}\right) \subset L^{2}\left(N ; d \mu_{g}\right) \text { is compact }
$$

for some $N \supset N(\operatorname{supp}(\chi) ; \epsilon)$ with some $\epsilon>0$, where $N(\operatorname{supp}(\chi) ; \epsilon)$ is the $\epsilon$ neighborhood of the support of $\chi$, then

$$
\sigma_{\mathrm{ess}}(\bar{\Delta}) \subset \sigma_{\mathrm{ess}}\left(\overline{\Delta^{\prime}}\right) .
$$

A special case of Theorem 1 is

Corollary 1 (Furutani's stability result 44). Let $g$ and $g^{\prime}$ be Riemannian metrics on $M$ such that $g=g^{\prime}$ outside a compact subset $K$ of $M$. If $\Delta$ is essentially self-adjoint in $L^{2}$, then $\Delta^{\prime}$ is essentially self-adjoint in $L^{2}\left(M ; d \mu_{g^{\prime}}\right)$, and

$$
\sigma_{\mathrm{ess}}(\bar{\Delta})=\sigma_{\mathrm{ess}}\left(\overline{\Delta^{\prime}}\right) .
$$

A typical example of manifolds which satisfies the condition of Theorem 1 is given as follows:

Corollary 2 (see Section 3). Let $M$ be a complete manifold and let $\Sigma \subset M$ be an almost polar compact subset. If $\Sigma$ is almost polar with respect to a metric $g^{\prime}$ on $M \backslash \Sigma$ and $g=g^{\prime}$ outside a compact set $K \subset M$, then the same conclusion in the theorem holds true.

We may apply Theorem 1 for singular manifolds: we change $g$ to $g^{\prime}$ on a bounded set $K \supset \partial_{C} M$ so that $g^{\prime}$ can be extended to the almost polar Cauchy boundary with respect to $g$ and conclude that $\Delta$ is essentially self-adjoint in $L^{2}\left(M ; d \mu_{g}\right)$ and $\sigma_{\text {ess }}(\bar{\Delta}) \subset \sigma_{\text {ess }}\left(\overline{\Delta^{\prime}}\right)$.

A sufficient condition for $\partial_{C} M$ to be almost polar is that it has Minkowski codimension greater than 2 [7] (if the metric of $g$ extends to $\partial_{C} M$ and $\partial_{C} M$ is a manifold, then it is almost polar if $\partial_{C} M$ has co-dimension 2).

The idea for proving the inclusion of the essential spectrum in Theorem 1 is to apply Weyl's criteria: a number $\lambda$ belongs to $\sigma_{e s s}(\bar{\Delta})$ if and only if there is a 
sequence $\phi_{n}$ of "limit-eigenfunctions" of $\bar{\Delta}$ corresponding to $\lambda$ (see Proposition 2 for details). Indeed, we show that if $\chi$ satisfies (2) and (3), then there is a subsequence $\phi_{n(k)}$ such that $(1-\chi) \phi_{n(k)}$ is a limit-eigenfunction of $\bar{\Delta}^{\prime}$.

Our results differ from Furutani's original results on the following two points. In order to explain those differences, let us employ an example. Let

$$
M=K \cup B(1),
$$

where $K=\left\{(x, y, z) \in S^{2}: z \geq 0\right\} \backslash(0,0,1)$ and $B(r)=\left\{(x, y, z) \in \mathbb{R}^{3}: x^{2}+y^{2} \leq\right.$ $\left.r^{2}, z=0\right\}$. Namely, $M$ is $S^{2}$ with flat bottom with deleted north point. (To be more precise, we need to smooth the intersection of $K$ and $B(1)$ so that $M$ is a smooth Riemannian manifold.) Since the Cauchy boundary of $M$ is the north point and it has null capacity, the Laplacian $\Delta$ is essentially self-adjoint. We modify $M$ by the stereographic projection so that $\left(M, g^{\prime}\right)$ is the 2-dimensional Euclidean space $\mathbb{R}^{2}$. Since $\left(M, g^{\prime}\right)$ is complete, the Cauchy boundary is empty, and the Laplacian $\Delta^{\prime}$ is essentially self-adjoint. Next, we find the function $\chi$ which satisfies condition (2) as follows:

$$
\chi \in C_{0}^{\infty}(M \backslash B(1 / 3)) \text { and } \chi=1 \text { on } K .
$$

By letting $\epsilon=1 / 3$ and $N=M \backslash B(1 / 4)$, condition (3) is satisfied. Indeed, since the north point has null capacity, the spectrum of the Laplacian on $M$ consists only of the eigenvalues with finite multiplicity, whereas $\Delta^{\prime}$ has only essential spectrum. This proves that the inclusion in Theorem 1 holds. This example also shows that the assumptions in Corollary 1 are sharp in the sense that we may not drop the assumption that $K$ must be compact in order to obtain (4), that is, Furutani's stability result. Indeed, if we modify the metric of $\mathbb{R}^{2}$ to obtain $M$, then there is no subset $N$ of $\mathbb{R}^{2}$ which satisfies condition (3).

The second difference is that the essential self-adjointness of $\Delta$ does not need to imply that of $\Delta$ restricted to $C_{0}^{\infty}(M)$; for instance, $\Delta$ on $M$ is essentially selfadjoint, but $\Delta$ restricted to $C_{0}^{\infty}(M)$ has infinitely many self-adjoint extensions. In particular, it is not essentially self-adjoint (see e.g. [2]).

We organize the article in the following manner: in Section 2] we prove Theorem 1, and in Section 3, we present the examples.

\section{ProOfS}

In this section we recall some definitions and prove Theorem 1 and Corollary 1 . For the sake of simplicity, we often suppress the symbols $M$ and $d \mu_{g}$.

We denote by $(\cdot, \cdot)$ and $(\cdot, \cdot)_{1}$ the inner product in $L^{2}$ and the Sobolev space $H^{1}$ of order $(1,2)$, respectively. $H_{0}^{1}$ is the completion of the set $C_{0}^{\infty}$ of smooth functions with compact support with respect to the norm $\|\cdot\|_{1}=\sqrt{(\cdot, \cdot)_{1}}$. Let $\mathcal{O}$ be the family of all open subsets of $\bar{M}$. For $A \in \mathcal{O}$ we define $\mathcal{L}_{A}=\left\{u \in H^{1}: u \geq\right.$ $1 \mu_{g}$-a.e. on $\left.M \cap A\right\}$,

$$
\operatorname{Cap}(A)= \begin{cases}\inf _{u \in \mathcal{L}_{A}}\|u\|_{1}, & \mathcal{L}_{A} \neq \phi \\ \infty, & \mathcal{L}_{A}=\phi\end{cases}
$$

and

$$
\operatorname{Cap}\left(\partial_{C} M\right)=\inf _{A \in \mathcal{O}, \partial_{C} M \subset A} \operatorname{Cap}(A)
$$


We will use

Proposition 1 (Lemma 2.1.1 of [3]). If $\mathcal{L}_{A} \neq \phi$ for $A \in \mathcal{O}$, there exists a unique element $e_{A} \in \mathcal{L}_{A}$ called the equilibrium potential of $A$ such that the following hold:

(i) $\left\|e_{A}\right\|_{1}^{2}=\operatorname{Cap}(A)$.

(ii) $0 \leq e_{A} \leq 1 \mu_{g}$-a.e. and $e_{A}=1 \mu_{g}$-a.e. on $A \cap M$.

(iii) If $A, B \in \mathcal{O}, A \subset B$, then $e_{A} \leq e_{B} \mu_{g}$-a.e.

We prove Theorem 1, We start from:

Proof of the essential self-adjointness of $\Delta^{\prime}$. For arbitrary $u \in H^{1}\left(M ; d \mu_{g^{\prime}}\right)$, we have to find $\hat{u}_{n} \in H^{1}\left(M ; d \mu_{g^{\prime}}\right)$ which converges to $u$ in $H^{1}\left(M ; d \mu_{g^{\prime}}\right)$. Indeed, this implies that $\left(M, g^{\prime}\right)$ has negligible boundary, and hence $\Delta^{\prime}$ is essentially self-adjoint by Gaffney's theorem [6].

Since $L^{\infty}(M) \cap H^{1}\left(M ; d \mu_{g^{\prime}}\right)$ is dense in $H^{1}\left(M ; d \mu_{g^{\prime}}\right)$, we may assume that $u \in$ $L^{\infty}(M)$ without loss of generality. Let

$$
\psi:=(1-r)_{+},
$$

where $r$ is the distance from $K$. The function $\psi \in L^{\infty}(M)$ enjoys the properties

$$
\left.\psi\right|_{K}=1 \quad \text { and } \quad\|\nabla \psi\|_{L^{\infty}} \leq 1 .
$$

Since

$$
|(1-\psi) u(x)| \leq\left(1+\|\psi\|_{L^{\infty}}\right)|u(x)|
$$

and

$$
|\nabla((1-\psi) u(x))| \leq\left(1+\|\psi\|_{L^{\infty}}\right)|\nabla u(x)|+|u(x)|,
$$

for almost every $x \in M$, it follows that $(1-\psi) u \in H^{1}$. Recalling that the essential self-adjointness of $\Delta$ implies $H_{0}^{1}=H^{1}[9]$, we find $v_{n} \in C_{0}^{\infty}(M \backslash K)$ such that

$$
v_{n} \rightarrow(1-\psi) u \text { as } n \rightarrow \infty
$$

in $H^{1}(M \backslash K)$. Because the Cauchy boundary $\partial_{C} M$ of $M$ associated to $g^{\prime}$ is almost polar, there is a sequence of the equilibrium potentials $e_{n}$ of $O_{n} \supset \partial_{C} M$ such that $\bigcap_{n>1} O_{n}=\partial_{C} M$ and

$$
\left\|e_{n}\right\|_{H^{1}\left(M ; d \mu_{g^{\prime}}\right)} \rightarrow 0 \text { as } n \rightarrow \infty .
$$

Then $u_{n}:=\left(1-e_{n}\right) \psi u \in H_{0}^{1}\left(M ; d \mu_{g^{\prime}}\right)$ satisfies

$$
u_{n} \rightarrow \psi u \text { as } n \rightarrow \infty
$$

in $H^{1}\left(M ; d \mu_{g^{\prime}}\right)$, and we get $\hat{u}_{n}=u_{n}+v_{n} \in H_{0}^{1}\left(M ; d \mu_{g^{\prime}}\right)$ such that

$$
\hat{u}_{n} \rightarrow(1-\psi) u+\psi u=u \text { as } n \rightarrow \infty
$$

in $H^{1}\left(M ; d \mu_{g^{\prime}}\right)$.

Next, we prove the inclusion of the essential spectrum and complete the proof of Theorem 1. We use the following characterization:

Proposition 2 (Weyl's criterion). A number $\lambda$ belongs to the essential spectrum of $\Delta$ if and only if there is a sequence of orthonormal vectors $\left\{\phi_{n}\right\}$ of $L^{2}$ such that

$$
\left\|(\Delta-\lambda) \phi_{n}\right\|_{L^{2}} \rightarrow 0 \text { as } n \rightarrow \infty .
$$


Proof of the inclusion of the essential spectrum. We assume (2) and (3) to prove the inclusion of the essential spectrum. Hereafter, we denote $\Delta=\bar{\Delta}$ and $\Delta^{\prime}=\overline{\Delta^{\prime}}$ because of their essential self-adjointness. Let $\lambda \in \sigma_{\mathrm{ess}}(\Delta)$ and $\phi_{n} \in D(\Delta)$ such that

$$
\begin{gathered}
\left(\phi_{i}, \phi_{j}\right)=\delta_{i j}, \\
\left\|(\Delta-\lambda) \phi_{n}\right\| \rightarrow 0 \text { as } n \rightarrow \infty,
\end{gathered}
$$

where $\|\cdot\|=\sqrt{(\cdot, \cdot)}$. Let $\chi$ be the function satisfying (2) and let $\phi$ be the function defined as

$$
\phi=(1-\hat{r} / \epsilon)_{+}
$$

where $\hat{r}$ is the distance from the support of $\chi$. Clearly, we have

$$
\sup _{n>0}\left\|\phi \phi_{n}\right\|<\infty \text {. }
$$

Moreover, taking into account that $\phi_{n} \in D(\Delta)$ implies

$$
\left\|\nabla \phi_{n}\right\|^{2}=-\left(\phi_{n}, \Delta \phi_{n}\right)
$$

it follows that

$$
\begin{aligned}
\limsup _{n \rightarrow \infty}\left\|\nabla\left(\phi \phi_{n}\right)\right\| & \leq\|\nabla \phi\|_{L^{\infty}}+\limsup _{n \rightarrow \infty}\left\|\nabla \phi_{n}\right\| \\
& \leq\|\nabla \phi\|_{L^{\infty}}+\limsup _{n \rightarrow \infty}\left(\left\|\Delta \phi_{n}\right\|\left\|\phi_{n}\right\|\right)^{1 / 2}<\infty
\end{aligned}
$$

Hence,

$$
\limsup _{n \rightarrow \infty}\left\|\phi \phi_{n}\right\|_{1}<\infty .
$$

Now, specifying $\epsilon>0$ as in the statement, by (7) and the fact that the embedding $H_{0}^{1}(N) \subset L^{2}(N)$ is compact, there exists a subsequence $\phi_{n(k)}$ of $\phi_{n}$ and $\phi^{\prime} \in L^{2}$ such that

$$
\phi \phi_{n(k)} \rightarrow \phi^{\prime} \text { strongly in } L^{2} \text { as } k \rightarrow \infty
$$

However, if $f \in L^{2}$, then $f \phi \in L^{2}$ and

$$
\left(f, \phi \phi_{n}\right)=\left(f \phi, \phi_{n}\right) \rightarrow 0 \text { as } n \rightarrow \infty
$$

hence, $\phi \phi_{n(k)} \rightarrow 0$ weakly in $L^{2}$ as $k \rightarrow \infty$. Because of the uniqueness of the weak-limits, it follows that $\phi^{\prime}=0$, and we may assume

$$
\left\|\phi \phi_{n}\right\| \rightarrow 0 \text { as } n \rightarrow \infty
$$

without loss of generality. Since $\phi=1$ on $\operatorname{supp}(\chi)$,

$$
\begin{aligned}
& \left\|(\Delta-\lambda)\left(\chi \phi_{n}\right)\right\| \\
& \leq\left\|(\Delta \chi) \phi \phi_{n}\right\|+2\left\|\left(\nabla \chi, \nabla \phi_{n}\right)\right\|+\left\|\chi(\Delta-\lambda) \phi_{n}\right\| \\
& \leq\|\Delta \chi\|_{L^{\infty}}\left\|\phi \phi_{n}\right\|+2\|\nabla \chi\|_{L^{\infty}}\left\|\nabla \phi_{n}\right\|_{L^{2}(\operatorname{supp}(\chi))}+\|\chi\|_{L^{\infty}}\left\|(\Delta-\lambda) \phi_{n}\right\| .
\end{aligned}
$$

The first and third terms in the last line tend to 0 as $n \rightarrow \infty$ because of (8) and (6). The second term can be estimated as

$$
\|\nabla \chi\|_{L^{\infty}}\left\|\nabla\left(\phi \phi_{n}\right)\right\| \leq\|\nabla \chi\|_{L^{\infty}} \sqrt{\left\|\Delta \phi_{n}\right\|\left\|\phi \phi_{n}\right\|} \rightarrow 0 \text { as } n \rightarrow \infty .
$$

Thus, since $1-\chi=0$ and $g=g^{\prime}$ on $M \backslash K$,

$$
\left\|\left(\Delta^{\prime}-\lambda\right)\left[(1-\chi) \phi_{n}\right]\right\|_{L^{2}\left(M ; d \mu_{g^{\prime}}\right)}=\left\|(\Delta-\lambda)\left[(1-\chi) \phi_{n}\right]\right\| \rightarrow 0 \text { as } n \rightarrow \infty .
$$


On the other hand,

$$
\left\|(1-\chi) \phi_{n}\right\|_{L^{2}\left(M ; d \mu_{g^{\prime}}\right)} \geq\left|\left\|\phi_{n}\right\|-\left\|\chi \phi_{n}\right\|\right| \geq\left|1-\left\|\phi \phi_{n}\right\|\right| \rightarrow 1 \text { as } n \rightarrow \infty,
$$

and we conclude that $\lambda \in \sigma_{\mathrm{ess}}\left(\Delta^{\prime}\right)$ by Weyl's criterion.

Finally, we assume that $K$ is compact to prove the essential self-adjointness of the Laplacian and the stability of the essential spectrum, namely, Corollary 1 .

Proof of Corollary 1. We will show that the Laplacian $\Delta^{\prime}$ is essentially self-adjoint and that there exist the function $\chi$ and the subset $N$ of $M$ which satisfy conditions (2) and (3) for each metric $g$ and $g^{\prime}$. This will imply

$$
\sigma_{\mathrm{ess}}(\bar{\Delta})=\sigma_{\mathrm{ess}}\left(\overline{\Delta^{\prime}}\right)
$$

by Theorem 1 .

Recall that if $K$ is compact, then its Cauchy boundary is empty so that the Laplacian $\Delta^{\prime}$ is essentially self-adjoint.

Since $K$ is compact and $g$ is smooth, there exists $\epsilon_{0}>0$ such that for any $0<\epsilon<\epsilon_{0}$, the metric $g$ and its higher order (up to 2nd) derivatives are bounded on $N=N(K ; 2 \epsilon)=\{x \in M: d(x, K)<2 \epsilon\}$. Let

$$
\hat{\chi}(x)=(1 \wedge(2-3 \tilde{r}(x) / \epsilon))_{+},
$$

where $\tilde{r}$ is the distance from $K$. The function $\hat{\chi}$ is 1 on $N(K ; \epsilon / 3)$ and has support in $N(K ; 2 \epsilon / 3)$, and it satisfies $\|\nabla \hat{\chi}\|_{L^{\infty}} \leq 3 / \epsilon$. However, since $\hat{\chi}$ does not need to be in the Sobolev space $H^{2}$ of order $(2,2)$, we apply the Friedrichs mollifier $j$ with radius $\delta>0$ for $\hat{\chi}$ to find the smooth function $\chi=j * \hat{\chi}$. If $\delta<\epsilon / 3$, then $\chi$ satisfies

$$
\left\{\begin{array}{l}
\chi(x)=1 \text { for } x \in K, \\
\operatorname{supp}(\chi) \subset N(K ; 2 \epsilon / 3), \\
\|\nabla \chi\|_{L^{\infty}} \leq 3 / \epsilon, \\
\Delta \chi \in L^{\infty},
\end{array}\right.
$$

namely, condition (2). On the other hand, since $K$ is compact, $N_{\epsilon}$ and $N$ are relatively compact in $M$ with sufficiently small $\epsilon>0$. Hence, $N$ has finite volume and finite diameter, and the Poincaré inequality holds on $N$. It follows that the embedding $H_{0}^{1}\left(N ; d \mu_{g}\right) \subset L^{2}\left(N ; d \mu_{g}\right)$ is compact, that is, condition (3)). We obtain the inclusion: $\sigma_{\mathrm{ess}}(\bar{\Delta}) \subset \sigma_{\mathrm{ess}}\left(\overline{\Delta^{\prime}}\right)$.

This argumentation holds true if we replace $g$ by $g^{\prime}$, and we arrive at the conclusion.

\section{EXAMPLES}

In this section, we present examples of manifolds for which Theorem 1 can be applied.

Example 1 (see [8]). Let $(M, g)$ be an $m$-dimensional complete Riemannian manifold and let $\Sigma \subset M$ be an $n$-dimensional compact manifold with $m \geq n+2$. Assume that $M$ has the product structure $M^{m-n} \times M^{n}$ near $\Sigma$ and that $g$ can be diagonalized. Choose local coordinates in a neighborhood $K$ of $\Sigma$ so that

$$
g=g_{1} \oplus g_{2}
$$


in $K$, where $g_{1}$ is a metric on $M^{m-n}$ and $g_{2}$ is a metric on $M^{n}$. Let $g^{\prime}$ be another smooth metric on $M \backslash \Sigma$ so that

$$
g^{\prime}= \begin{cases}f^{2} g_{1} \oplus g_{2}, & \text { on } K, \\ g, & \text { on } M \backslash K .\end{cases}
$$

If $m=2$, assume that $f \in L^{2+\epsilon}\left(K ; d \mu_{g}\right)$ for some $\epsilon \in(0, \infty)$.

If $m=3$, assume that $\inf (f)>0$ and $f \in L^{(m(m-2) / 2)+\epsilon}\left(K ; d \mu_{g}\right)$ for some $\epsilon \in(0, \infty)$.

Then the manifold $M \backslash \Sigma$ with metrics $g$ and $g^{\prime}$ satisfies the assumption of Theorem 1. In particular, if $M$ is compact, $\Delta^{\prime}$ on $\left(M \backslash \Sigma, g^{\prime}\right)$ has discrete spectrum, which satisfies the Weyl asymptotic formula [8].

In the next example, the manifold has fractal singularity.

Example 2. Let $(M, g)$ be a complete Riemannian manifold with dimension greater than 2. Let $\Sigma \subset M$ be the Cantor set, $r$ the distance in $M$ from $\Sigma$, and $B(R)$ the $R$-neighborhood of $\Sigma$. Set

$$
g^{\prime}=f^{2} g
$$

where

$$
f(x)= \begin{cases}r^{\epsilon}, & x \in B=B(1), \\ 1, & x \in M \backslash B .\end{cases}
$$

It is shown in [9] that $\Sigma$ is the almost polar Cauchy boundary of $\left(M \backslash \Sigma ; g^{\prime}\right)$ if

$$
\epsilon>\frac{\ln 2-\ln 3}{2 \ln 3-\ln 2} \text {. }
$$

The compact inclusion

$$
H_{0}^{1}(B \backslash \Sigma) \subset L^{2}(B \backslash \Sigma)
$$

can be seen as follows. By definition, $H_{0}^{1}(B \backslash \Sigma) \subset H_{0}^{1}(B)$, and the inclusion $H_{0}^{1}(B) \subset L^{2}(B)=L^{2}(B \backslash \Sigma)$ is compact; thus, it suffices to show

$$
H_{0}^{1}(B \backslash \Sigma) \supset H_{0}^{1}(B) .
$$

Let $u \in H_{0}^{1}(B)$. Since $L^{\infty} \cap H_{0}^{1}(B) \subset H_{0}^{1}(B)$ is dense, we may assume that $u \in L^{\infty}$ without loss of generality. Let $e_{n}$ be the equilibrium potential as in the proof of Theorem 1 Then $u_{n}=u\left(1-e_{n}\right) \in H_{0}^{1}(B \backslash \Sigma)$ and

$$
u_{n} \rightarrow u \text { in } H_{0}^{1}\left(B ; d \mu_{g}\right),
$$

and hence $u \in H_{0}^{1}(B \backslash \Sigma)$. The function $\chi$ can be found as the relative equilibrium potential of $B(1)$ and $B(2)$ applied to the Friedrichs mollifier. Therefore, $M \backslash \Sigma$ together with $g$ and $g^{\prime}$ satisfies the condition of Theorem 1. Let us point out the following:

- $\left(M \backslash \Sigma, g^{\prime}\right)$ is $C^{1,1}$ and is not smooth, but Theorem 1 can be applied to this setting.

- We can show the compact embedding $H_{0}^{1}\left(B \backslash \Sigma ; d \mu_{g^{\prime}}\right) \subset L^{2}\left(B ; \backslash \Sigma ; d \mu_{g^{\prime}}\right)$ only for $\epsilon \geq 0$.

In the next example, $K$ has infinite volume with $g^{\prime}$. 
Example 3. Let $M$ be a 2-dimensional complete Riemannian manifold. Delete a point $p \in M$ and set

$$
g^{\prime}=f^{2} g,
$$

where

$$
f(x)= \begin{cases}r^{-\epsilon}, & x \in B=B(1), \\ 1, & x \in M \backslash B\end{cases}
$$

and $r$ is the distance from $p$. For any $\epsilon \geq 1,\left(M \backslash\{p\}, g^{\prime}\right)$ is complete and $\mu_{g^{\prime}}(B \backslash\{p\})=\infty$.

More generally, if $M$ is a complete manifold and $\Sigma \subset M$ is a compact set, then there is a smooth Riemannian metric $g^{\prime}$ on $M \backslash \Sigma$ and a compact set $K \subset M$ such that $g=g^{\prime}$ on $M \backslash K,\left(M \backslash \Sigma ; g^{\prime}\right)$ is complete and there exist a function $\chi$ and a subset $N$ of $M \backslash \Sigma$ satisfying conditions (2) and (3), respectively.

\section{ACKNOWLEDGMENT}

The author would like to thank the referee for a careful reading and constructive discussions.

\section{REFERENCES}

[1] Bando, S.; and Urakawa, H., Generic properties of the eigenvalue of the Laplacian for compact Riemannian manifolds. Tohoku Math. J. (2) 35 (1983), no. 2, 155-172. MR699924(84h:58146)

[2] Colin de Verdière, Yves, Pseudo-laplaciens. I. Ann. Inst. Fourier (Grenoble) 32 (1982), no. 3, xiii, 275-286. MR688031 (84k:58221)

[3] Fukushima, M; Oshima, Y.; Takeda, M., Dirichlet forms and symmetric Markov processes. de Gruyter Studies in Mathematics, 19. Walter de Gruyter \& Co., Berlin, 1994. MR.1303354 (96f:60126)

[4] Furutani, K., On a stability of essential spectra of Laplace operators on non-compact Riemannian manifolds. Proc. Japan Acad. 56, Ser. A (1980), 425-428. MR603058 (82c:58055)

[5] Gaffney, M.P., A special Stokes's theorem for complete Riemannian manifolds. Ann. of Math. (2) 60 (1954), 140-145. MR0062490 (15:986d)

[6] Gaffney, M.P., Hilbert space methods in the theory of harmonic integrals. Trans. Amer. Math. Soc. 78 (1955), 426-444. MR0068888 (16:957a)

[7] Masamune, J., Essential self-adjointness of Laplacians on Riemannian manifolds with fractal boundary. Comm. Partial Differential Equations 24 (1999), no. 3-4, 749-757. MR1683058 (2000m:58035)

[8] Masamune, J.; Rossman, W., Discrete spectrum and Weyl's asymptotic formula for incomplete manifolds. Minimal surfaces, geometric analysis and symplectic geometry (Baltimore, MD, 1999), 219-229, Adv. Stud. Pure Math., 34, Math. Soc. Japan, Tokyo, 2002. MR1925741 (2004b:58052)

[9] Masamune, J., Analysis of the Laplacian of an incomplete manifold with almost polar boundary. Rend. Mat. Appl. (7) 25 (2005), no. 1, 109-126. MR2142127 (2006a:58040)

Department of Mathematical Sciences, Worcester Polytechnic Institute, 100 Institute Road, Worcester, Massachusetts 01609-2280

Current address: Department of Mathematics and Statistics, Pennsylvania State UniversityAltoona, 3000 Ivyside Park, Altoona, Pennsylvania 16601

E-mail address: jum35@psu.edu 